\title{
BMJ Open Combating antibiotic resistance using guidelines and enhanced stewardship in Kenya: a protocol for an implementation science approach
}

\author{
Jesse Gitaka, ${ }^{1}$ Moses Kamita (D) , ${ }^{1}$ Dominic Mureithi, ${ }^{2}$ Davies Ndegwa, ${ }^{3}$ \\ Moses Masika, ${ }^{4}$ Geoffrey Omuse, ${ }^{5}$ Moses Ngari, ${ }^{6}$ Francis Makokha, ${ }^{1}$ \\ Peter Mwaura, ${ }^{1}$ Ronald Mathai, ${ }^{1}$ Francis Muregi, ${ }^{1}$ Matilu Mwau ${ }^{7}$
}

To cite: Gitaka J, Kamita M, Mureithi D, et al. Combating antibiotic resistance using guidelines and enhanced stewardship in Kenya: a protocol for an implementation science approach. BMJ Open 2020;10:e030823. doi:10.1136/ bmjopen-2019-030823

- Prepublication history and additional material for this paper are available online. To view these files, please visit the journal online (http://dx.doi. org/10.1136/bmjopen-2019030823).

Received 04 April 2019 Revised 17 December 2019 Accepted 06 March 2020

Check for updates

(C) Author(s) (or their employer(s)) 2020. Re-use permitted under CC BY-NC. No commercial re-use. See rights and permissions. Published by BMJ.

For numbered affiliations see end of article.

Correspondence to

Dr Jesse Gitaka;

jgitaka@mku.ac.ke

\section{ABSTRACT}

Introduction Antimicrobial resistance (AMR) is a growing problem globally especially in Sub-Saharan Africa including Kenya. Without any intervention, lower/middle-income countries (LMICs) will be most affected due to already higher AMR levels compared with higher income countries and due to the far higher burden of diseases in the LMICs. Studies have consistently shown that inappropriate use of antimicrobials is the major driver of AMR. To address this challenge, hospitals are now implementing antibiotic stewardship programmes (ASPs), which have been shown to achieve reduced antibiotic usage, to decrease the prevalence of resistance and lead to significant economic benefits. However, the implementation of the guideline is highly dependent on the settings in which they are rolled out. This study, employing an implementation science approach, aims to address the knowledge gap in this area and provide critical data as well as practical experiences when using antibiotic guidelines and stewardship programmes in the public health sector. This will provide evidence of ASP performance and potentially contribute to the county, national and regional policies on antibiotics use.

Methods and analysis The study will be conducted in three geographically diverse regions, each represented by two hospitals. A baseline study on antibiotic usage, resistance and de-escalation, duration of hospital stay, rates of readmission and costs will be carried out in the preimplementation phase. The intervention, that is, the use of antibiotic guidelines and ASPs will be instituted for 18 months using a stepwise implementation strategy that will facilitate learning and continuous improvement of stewardship activities and updating of guidelines to reflect the evolving antibiotic needs.

Ethics and dissemination Approvals to carry out the study have been obtained from the National Commission for Science, Technology and Innovation and the Mount Kenya University Ethics Review Committee. The approvals from the two institutions were used to obtain permission to conduct the study at each of the participating hospitals. Study findings will be presented to policy stakeholders and published in peer-reviewed scientific journals. It is anticipated that the findings will inform the appropriate antibiotic use guidelines within our local context.
Strengths and limitations of this study

- First study aimed at concurrently rolling out antimicrobial stewardship committees in multiple hospitals in Kenya.

- Use of implementation approach to support implementation of suggested guidelines for antimicrobial resistance surveillance.

- First-hand evidence on the antimicrobial resistance in three diverse counties in Kenya.

- The study is limited to only 3 of the 47 counties in Kenya.

\section{INTRODUCTION}

Antibiotic resistance is a serious public health concern globally ${ }^{12}$ and locally ${ }^{34}$ and fears of running out of antibiotic options in the near future have been expressed. In 2016, WHO called for immediate and concerted efforts to mitigate this threat to global health, which was estimated to contribute to 700000 deaths in 2014 and projected to cause 10 million deaths in 2050, if inadequately mitigated. ${ }^{5}$ The driving force escalating rates of resistance is the injudicious use of antibiotics in patients, in livestock and in agriculture, and its unchecked release into the environment. ${ }^{6}$ These forces raise and increase the spread of resistant pathogens that may emerge by mutations or acquisition of plasmids carrying resistance genes. ${ }^{7}$ In one study, prior antibiotic exposure was the key independent risk factor for the acquisition of antibiotic multiresistance. ${ }^{8}$ Broad-spectrum antibiotics have the unintended consequence of selecting multidrug-resistant pathogens and increasing the likelihood of infection by fungi and Clostridium difficile. $^{9}$

The injudicious use of antibiotics is widespread. In Africa, many patients do not receive treatment from the conventional healthcare 
system. Of those who receive antibiotics, $31.7 \%$ do not consult a doctor for a prescription and a further $26.4 \%$ obtain the antibiotics over the counter. ${ }^{10}$ A study in South Africa found that $54.9 \%$ of antibiotics were inappropriately prescribed in intensive care unit settings while in the USA, $20 \%-50 \%$ of prescribed antibiotics were unnecessary or unwarranted. ${ }^{11-13}$

The rate of antimicrobial resistance in developing countries like Kenya is worrying and rising. ${ }^{14}$ For example, the prevalence of Salmonella typhi resistant to two or more antimicrobials was found to have increased from $50 \%$ in 1998 to $78 \%$ in 2004 at Kenyatta National Hospital. ${ }^{15}$ The Global Antibiotic Resistance Partnership (GARP) — Kenya Working Group Report of 2011 identified antibiotic resistance as a key issue in Kenya and made recommendations to curtail the spread. ${ }^{16}$ These recommendations included the use of antibiotic guidelines that took into consideration local resistance surveillance data and enhanced antibiotic stewardship programmes (ASPs). ${ }^{16}$ However, these ASPs have not been instituted at county hospitals, and key implementation data and experiences are lacking in their roll out.

Antibiotic stewardship is defined as 'the optimum selection, dosage, and duration of antimicrobial treatment that yields the best clinical outcomes for the treatment or prevention of infection with the least toxicity to the patient and minimal impact on subsequent resistance'. ${ }^{17}$ It has the potential to lower treatment cost and realise economic benefits to the patient, healthcare system and the country at large. ${ }^{18} 19$ Moreover, optimising antibiotic use by minimising exposure, fine-tuning dosage and reducing superfluous therapy and focusing treatment to the likely culprit pathogens is a strategy that boosts patient safety ${ }^{20}$ and ultimately safeguards against antibiotic resistance. ${ }^{21}$

\section{JUSTIFICATION}

Antibiotic resistance is a major health challenge globally. Studies in Kenya have shown rising antibiotic resistance over the last three decades. While antibiotic guidelines and ASPs have been observed to lead to significant economic benefits, reduce antibiotic usage and lower the prevalence of resistance especially in Europe, North America, Japan and South Africa, they have not been employed to tackle the challenge in the public health sector in Kenya. The GARP report of 2011 recognised the use of guidelines and stewardship programmes as a potential strategy in 'saving antibiotics' but noted the need for local studies to understand implementation challenges, derive lessons and embed the strategy within the Kenyan healthcare system. The here proposed study, employing an implementation science approach, aims to address the knowledge and research gap in this area and provide critical data and experiences in using antibiotic guidelines and stewardship programmes in the public health sector.
OBJECTIVES OF THE STUDY

\section{General objectives}

The overall aim is to evaluate the impact of antibiotic guidelines for empirical treatment of (1) urinary tract infections, (2) community-acquired pneumonia, (3) bacteraemia and (4) meningitis in terms of reducing usage of broad-spectrum antibiotics, antibiotic de-escalation, duration of hospital stays, rates of readmission and prevalence of antibiotic resistance in six county hospitals in Kenya.

\section{Specific objectives}

1. To develop guidelines for antibiotic use for common infections.

2. To set up antibiotic steward committees (ASCs) in six county hospitals.

3. To train the ASC members such that they can fully perform their mandate.

4. To measure the usage of broad-spectrum antibiotics, antibiotic de-escalation, duration of hospital stays and rates of readmission in hospitals using the antibiotic stewardship strategy.

5. To ascertain antibiotic resistance patterns using culture, sensitivity and genetic markers.

6. To establish the knowledge of healthcare workers, their attitudes and prescription practices regarding antibiotics resistance, use of guidelines and ASPs.

7. To evaluate the economic benefits of using guidelines for antibiotics use and ASPs.

\section{Expected outputs of the research}

1. Antibiotic guidelines informed by local bacteria susceptibility patterns.

2. ASCs in six county hospitals in Kenya.

3. Trained ASC members.

4. Data on antibiotics usage, antibiotic de-escalation, duration of hospital stay and rates of readmission in hospitals using the antibiotic stewardship strategy.

5. Map and data of antibiotic resistance patterns before and after implementation of the strategy.

6. Qualitative data on the knowledge of healthcare workers, their attitudes and practices regarding antibiotic usage and stewardship programmes.

7. Publication on cost-benefit analysis (CBA) for using antibiotic guidelines and ASPs.

\section{METHODS AND ANALYSIS}

\section{Setting}

To be able to evaluate the antibiotic stewardship strategy across Kenya using an implementation science approach, the study will be conducted in six county hospitals, namely (1) Kiambu County Referral Hospital, (2) Bungoma County Hospital, (3) Webuye Sub-County Hospital, (4) Nakuru County Teaching and Referral Hospital, (5) Thika Level 5 Hospital and (6) Naivasha Sub-County Hospital. 


\section{Design}

The study will use the Reach, Effectiveness, Adoption, Implementation and Maintenance (RE-AIM) conceptual framework. RE-AIM involves the interaction of all the five factors and fits well with approaches that are system based. ${ }^{22}$ The study design will encompass a preimplementation phase, a stepwise implementation phase and an endline study to measure the changes in outcomes between the phases. A baseline study on antibiotic usage (defined daily doses (DDD) per 1000), antibiotic resistance (culture and genetic markers), antibiotic de-escalation, duration of hospital stays, rates of readmission, prescription patterns and cost analysis will be carried out in the preimplementation phase. The intervention, which is the use of antibiotic guidelines and ASPs, will be instituted in the 6 county hospitals for 18 months. The stepwise implementation phase will involve introduction of interventions as well as monitoring and evaluation of their effectiveness, and thereafter improving the interventions where necessary. The strategy will facilitate learning cycles every 4-6 weeks and continuous improvement of stewardship activities and updating of guidelines to reflect evolving antibiotic needs in diverse settings. During the intervention phase, challenges faced during the implementation of the guidelines will be documented and used to advise on the changes required during the review of the guidelines. The endline study will be conducted and differences between the two phases evaluated as per statistical analysis.

\section{In the baseline and endline studies, multidisciplinary strategies will be employed as follows}

1. Knowledge of healthcare workers, attitudes and practices on antibiotic resistance, guidelines and ASP will be studied qualitatively and quantitatively.

2. Basic science approaches, encompassing antibiotics culture sensitivity and molecular biology-genetic markers of resistance, will be analysed.

3. Clinical patient outcomes will be studied to evaluate the guidelines.

4. Health economics cost savings on using guidelines and ASP will be evaluated.

\section{Antibiotic guidelines and ASPs: development}

Antibiotic guidelines will be formulated in consultation with senior clinicians in the study hospitals taking into account each hospital's antimicrobial resistance patterns. The ASP committee will comprise a hospital physician, a microbiologist and a pharmacist. Broad-spectrum antibiotic prescriptions will be brought to the attention of the ASP committee members. The committee members will perform regular ward rounds, three times a week, in the initial stages. This will be reduced to once a week later in the project, to optimise adherence to antibiotic guidelines. Furthermore, the guidelines will be promoted through training sessions, provision of pocket-size guideline cards to clinicians and pharmacists, large poster displays in the wards and through hospital and project websites and social media.

\section{Data collection}

Laboratory assessment tools will be used to determine the preparedness of the laboratories to perform antibiotic sensitivity tests (see online supplementary file 1 ). The tool will assess whether the laboratories have the equipment necessary to perform bacterial culture, identification and susceptibility testing, such as carbon dioxide incubators, safety cabinets and refrigerators. The tool will also determine whether the laboratories have the internationally recommended guidelines to perform susceptibility and quality assurance tests. In addition, knowledge, attitude and practices (KAPs) about antibiotic prescription and resistance among medical practitioners will be assessed using a KAP tool (see online supplementary file 2). The tool will target those medical practitioners, who usually prescribe antimicrobial drugs in the hospitals and will include the consultants, medical officers as well as interns, clinical officers and pharmacists. The KAP tool will also determine the guidelines that the prescribers use to decide on the appropriate antimicrobial drug. A health system assessment tool will be employed to gather baseline information on the antimicrobial stewardship activities in each hospital (see online supplementary file 3). Information on bed occupancy, antibiotic usage and antibiotic resistance data will be collected from each of the hospital information systems, pharmacy management systems and laboratory reporting systems, respectively. Antibiotic usage will be analysed in order to determine the impact of the intervention on antibiotic usage comparing the intervention and the control arms. Data on antibiotics issued to adult and children inpatients only will be factored, excluding discharge and outpatient supplies.

To consistently compare antibiotic usage, the DDD will be used and expressed per 1000 occupied bed days (OBDs) to account for fluctuations in activity following WHO guidelines. ${ }^{23}$ The OBDs will be obtained from the hospital information systems.

\section{Culture sensitivity and genetic analyses}

Nasal swabs or tracheal aspirate, urine, wound swabs and blood samples will be taken from patients who have consented to the study for bacteriological analysis. Samples will be subjected to standard bacteriological analysis to isolate the culprit bacteria. Bacterial species will be confirmed by use of biochemical test and analytical profiles index API strips (bioMérieux France). Antimicrobial susceptibility tests will be performed on isolated bacteria as per the Kirby-Bauer method following manufacturer's instruction. Results will be interpreted using the Clinical and Laboratory Standards Institute tables. ${ }^{24}$

Any bacteria isolate found to be resistant to third generation cephalosporins will be tested for production of extended spectrum beta-lactamase (ESBL) using the synergy disk diffusion test. Vancomycin-resistant Enterococci (VRE) will be identified using disc diffusion tests. Methicillin-resistant Staphylococcus aureus (MRSA) will be detected by testing isolates resistant to cefoxitin using the E test (AB Biodisk, Solna, Sweden) on Mueller-Hinton 
agar supplemented with $2 \% \mathrm{NaCl}$ and incubated at $37^{\circ} \mathrm{C}$ for 24 hours. The identified ESBL, VRE and MRSA samples will be analysed by PCR and sequencing to identify the resistance genotype. In vitro conjugation tests will be performed to determine if resistance in bacteria is transferable.

\section{CBA for the use of antibiotic guidelines and ASP}

A CBA from a health facility and a national perspective will be performed. For health facilities, three cost drivers will be considered: (1) pharmacy spending, (2) length of stay and (3) antimicrobial stewardship interventions (training, infection control measures and so on). At country level, we will consider disability-adjusted life years, work-days lost and cost of treatment. ${ }^{25-27}$ This will be done by collecting and analysing data on patient income, length of hospital stay, death or disability occasioned by drug-resistant pathogens, hospital pharmacy expenditure and cost of training/rolling out antimicrobial stewardship guidelines. This data will be collected before and after antimicrobial stewardship interventions.

\section{Study size}

Prevalence of inappropriate use of broad-spectrum antibiotics

The sample size is based on the prevalence of inappropriate use of broad-spectrum antibiotics, documented as being about $50 \%$ in South Africa. ${ }^{11}$ To detect a reduction of the $50 \%$ inappropriate use of broad-spectrum antibiotics by $20 \%-30 \%$ with a power of $90 \%$ and $\alpha$ of 0.05 in a two-sided test, a sample size of 410 in each county hospital would be adequate. In order to detect a reduction (20\%) but with a power $80 \%$, the sample size would be 320 as shown in figure 1 . With a $15 \%$ allowance for loss to follow-up, a total of about 500 participants would be sufficient in each of the selected county hospitals. Therefore, we shall evaluate the prevalence of inappropriate use of broad-spectrum antibiotics in $500 \times 6=3000$ patients from the six facilities.

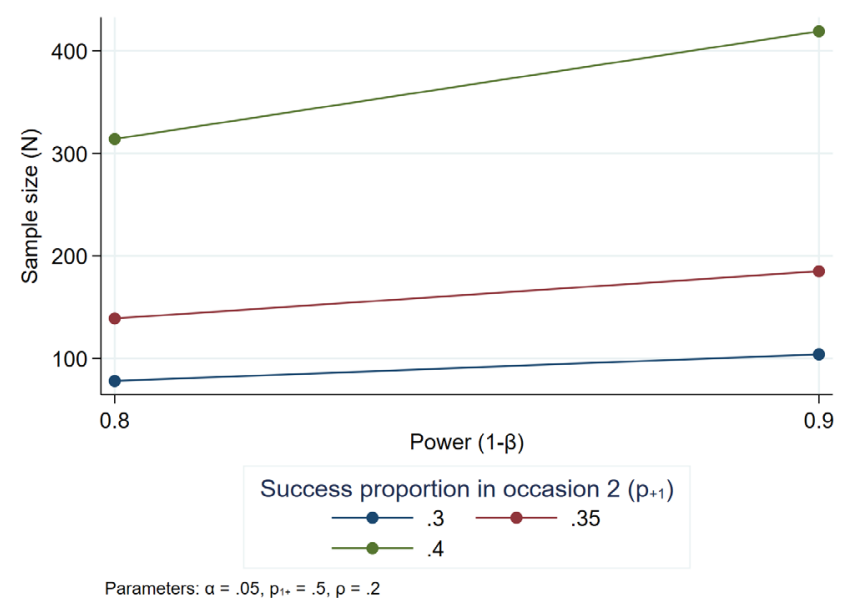

Figure 1 Statistical power analysis for sample size determination for the prevalence of inappropriate use of broad-spectrum antibiotics.

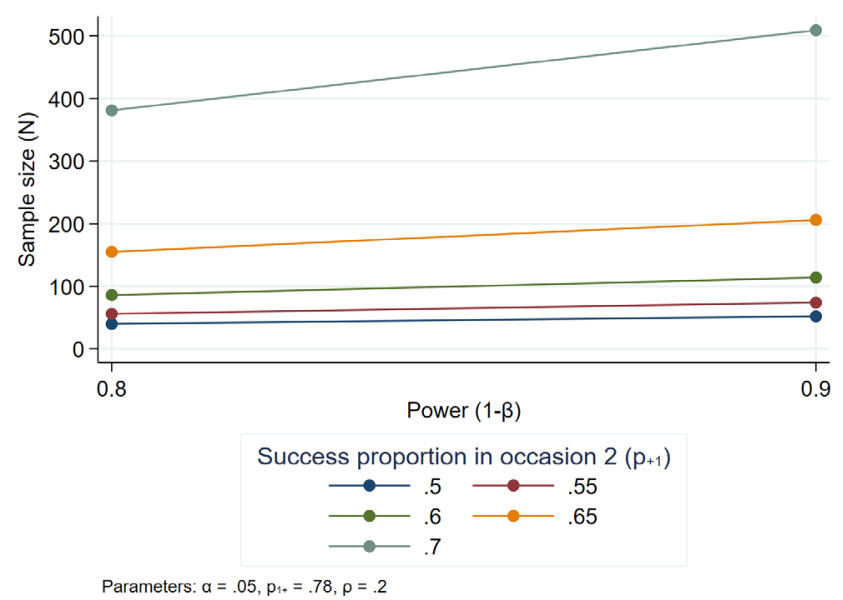

Figure 2 Statistical power analysis for sample size determination for the prevalence of antibiotics resistance.

\section{Prevalence of antibiotic resistance}

The sample size is based on the prevalence of antibiotic resistance of $78 \%{ }^{15}$ in 2004 at Kenyatta National Hospital, Kenya. To detect a reduction of the $78 \%$ antibiotic resistance by $10 \%-68 \%$ with a power of $90 \%$ and $\alpha$ of 0.05 two-sided test, a sample size of 500 in each county hospital would be adequate; while to achieve a reduction of $10 \%$ with power $80 \%$, the sample size would need to be 220 (figure 2). With $15 \%$ allowance for loss to follow-up, a total of 600 participants would be sufficient in each of the selected county hospitals. Therefore, we shall evaluate the prevalence of resistance in $600 \times 6=3600$ patients for the 6 facilities.

\section{Data management}

Data will be recorded on a standardised case report form at every hospital by the trained study staff. The data will be held locally and uploaded to the secure central study server hosted at the Mount Kenya University main campus in Thika. This will be overseen by the data manager/ statistician, who will run regular reconciliation to derive the final study database. Access to the study database will be restricted and password protected. The data will be stored in excel, jpeg and word files for the electronic cases. For archiving purposes, the Mount Kenya University electronic repository will be used to ensure that the data are publicly available. Collected data will be retained according to the policy governing the Mount Kenya University repository. The principal investigators involved in this study do not have any reasons that might prohibit the sharing of the data emanating from the study.

\section{Statistical analysis}

The data will be analysed using qualitative and quantitative methods. Qualitative data will be analysed by subjecting the information to content analysis and presenting it in different emerging themes. The summaries of the data emanating from these themes will then be arranged on a case-by-case basis through the use of an Excel spreadsheet ${ }^{28}$ and the analyses performed using 
NVivo software. Quantitative data analyses will be carried out using Stata V.14 (Stata). The differences between baseline and endline on all the study outcomes will be compared using appropriate statistical methods, such as McNemar's test, paired t-test and the Wilcoxon signedrank test (non-parametric test), accounting for pairing and clusters (hospitals). Multivariable log-binomial regression analysis will be used to elucidate risk factors for antibiotic resistance.

\section{ETHICS AND DISSEMINATION}

Approvals to carry out the study have been obtained from the National Commission for Science, Technology and Innovation (NACOSTI) (NACOSTI/P/18/33304/25986) and the Mount Kenya University Ethics Review Committee (MKU/ERC/0764). In addition, permission was obtained from each of the participating hospitals. This entailed submission of an introduction letter attaching the certificate of clearance from NACOSTI and the ethical review certificate from MKU. In Thika, Nakuru and Naivasha, a research fee was paid as required by the hospital board. The study will follow all provisions of the Declaration of Helsinki. Participants in the study will not incur any cost associated with the transport or processing of the samples; neither will they receive any monetary inducements to participate in the study. Material transfer to laboratories outside of Kenya shall not be undertaken in this study. Informed written consent will be sought from the participants enrolled in the study.

Obtained results will be disseminated via different streams, including research conferences and in peerreviewed journals. We are aiming for five publications to be generated by the end of the study. These will include the study protocol itself and the different aspects emerging during in the course of the project. Contextualised guidelines on judicious use of antibiotics in the six hospitals in Kenya will also be published in the Ministry of Health's website and that of the collaborating hospitals for easy access by other health facility in and outside Kenya. In addition, the findings will be shared in a dissemination forum bring together members of the health management teams at both the country and county levels, clinicians who do prescription of antimicrobial drugs, researchers, members of the public and other key stakeholders. The dissemination forum will be held once at the end of the study.

\section{Patient and public involvement}

The study is mainly targeting clinicians who do antimicrobial proscription and, therefore, patients will not be directly involved in the study. Reports obtained from this study will, however, be made available to the patients in the participating hospitals through a dissemination forum and to the general public through publications.

\section{Exit strategy and stakeholder involvement}

In the process of developing this protocol, we have engaged physicians working in the proposed county hospitals, and they have identified the challenge of antibiotic resistance as real and appreciate the opportunities that use of antibiotic guidelines and ASPs may provide in combating resistance, improving clinical care and saving costs. We plan to continue involving all the stakeholders in the process, including clinicians, laboratory personnel, pharmacists, public health officials, patients and scientists. We anticipate that the study findings will inform county and national policy on mitigating antibiotic resistance and raise public awareness on the need for judicious use of antibiotics. The project will use social media platforms, websites of the collaborating institutions and publications in peer-reviewed journals, local dailies and presentations in scientific meetings to further engage stakeholders and the public on this important issue and enhance the learning approach inherent in the strategy of implementation science for improved performance of the ASPs and the study in general. In case any of the hospitals dropout either during the baseline phase or early stages of the implementation phase, another hospital will be brought onboard. Replacements will not be considered during the late stages of the project.

\section{DISCUSSION}

The emergence of antimicrobial-resistant pathogens and a lack of new drugs to effectively treat these pathogens are the two main challenges in human health. There is thus the need to advocate the proper use of the currently available antimicrobial agents by safeguarding their effectiveness. Antibiotic stewardship has been shown to contribute to reducing antibiotic resistance, ${ }^{29}{ }^{30}$ but this strategy has not been rolled out in most sub-Saharan countries in level 4 and 5 hospitals. The proposed work will employ an implementation research approach to evaluate the best strategies and derive lessons on mainstreaming antibiotic stewardship in these facilities. By leveraging on a health system approach, the implementation research will unravel real-life impediments and opportunities by working with hospital teams to codesign execution plans, monitor and evaluate and the sustainability of the stewardship programmes.

Most hospitals in Kenya lack the capacity to carry out antimicrobial sensitivity tests, due to the lack of resources and technical knowhow, among other challenges. ${ }^{31}$ This study will first provide an assessment to determine the challenges hospitals are facing; this will be done through interviews with clinicians and assessment of the capacity of the laboratories to perform the sensitivity tests. These steps will make up the initial phase and will guide the nature of implementations to be used during the implementation phase. It is hoped that the project will capacitate the laboratories in the six hospitals to do the tests and sensitise the clinicians on the need to prescribe antimicrobial drugs based on results obtained from the laboratory tests. After the implementation phase, the endline phase will involve surveys similar to those conducted during the baseline phase. This will allow determination 
Table 1 Proposed study timelines

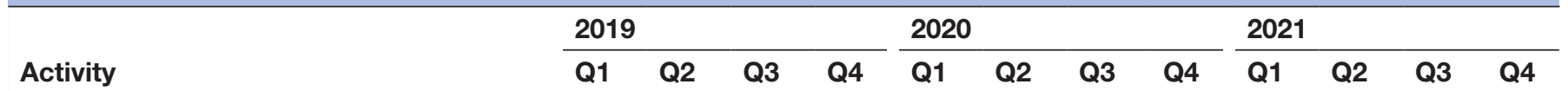

Ethics clearance and authorisation from study sites

Baseline study on prevalence of antibiotic resistance and stewardship activities

Identification of members for the antibiotic stewardship programmes (ASPs)

Development of antibiotic guidelines

Adopting and preparing materials for ASP training

Development of antibiotic guidelines and stewardship mobile application

Training the ASP members

Collecting data on antibiotic usage in the hospitals

Conducting KAPs study on use of guidelines and ASPs

Analyse and present antibiotic resistance

data using maps and charts

Presenting findings to stakeholders and preparing the final report

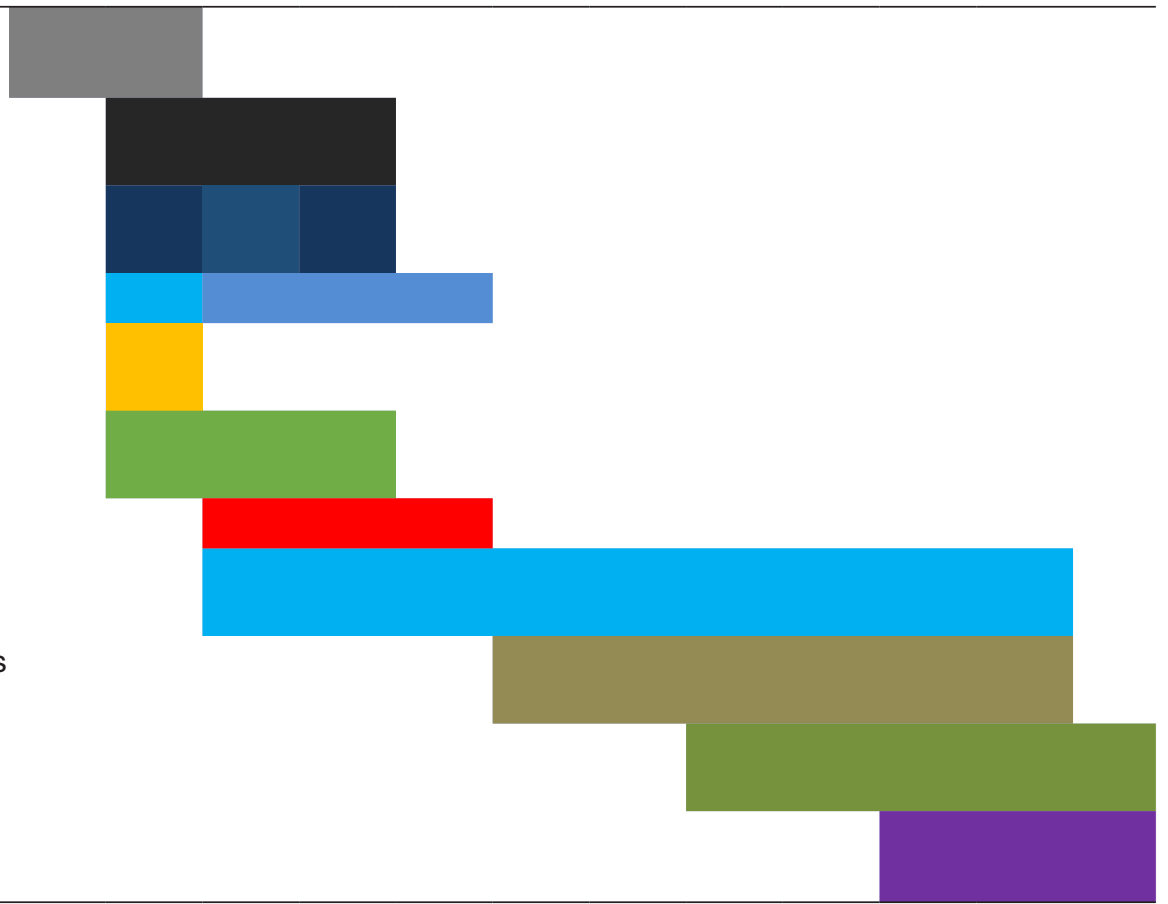

KAP, knowledge, attitude and practices.

of the impact of the implementation strategies taken in reducing the cost of treatment, length of hospital stay and burden on the health system, among others.

The hospital management teams play a key role in determining the allocation of resources to and within the hospitals. ${ }^{32}$ In this project, we have proposed to involve hospital management by including its members in the stewardship committees that will be established. This will ensure that the management is well informed about the challenges in the sections involved in surveillance and the progress that is being made. Involvement of the hospital management will also ensure that there is a buy-in of the recommendations made by the stewardship committee.

Although there is the need to establish antimicrobial stewardship committees in all the hospitals, the project proposes to start with the selected six hospitals with the hope that the same can be reproduced by other hospitals to establish similar committees in their hospitals.

\section{Study timeline}

The study is designed for a 3-year period. Enrolment already started in 2018 and participant recruitment will continue up to 2020 (table 1). So far, stewardship committees have been established and workshops to sensitise the members conducted. Data collection on KAP of antibiotic prescribers in the hospitals is already in progress.

Author affiliations

${ }^{1}$ Research and Innovation, Mount Kenya University, Thika, Kenya
${ }^{2}$ Department of Animal Health and Production, Maasai Mara University, Narok, Kenya

${ }^{3}$ Department of Medical Laboratory Sciences, Kenya Medical Training College, Nairobi, Kenya

${ }^{4}$ Department of Medical Microbiology, University of Nairobi College of Health Sciences, Nairobi, Kenya

${ }^{5}$ Department of Pathology, Aga Khan University, Nairobi, Kenya

${ }^{6}$ Clinical Trial Facility, KEMRI/Wellcome Trust, Kilifi, Kenya

${ }^{7}$ Centre for Infectious and Parasitic Diseases Control Research, Kenya Medical Research Institute, Nairobi, Kenya

Contributors JG initiated the concepts of the study. JG, DM, DN, MMasika, GO, MN, FMakokha, PM, RM, FMuregi and MMwau developed the protocol. JG, DM, DN and MMasika will be involved in training the ASCs. DM, GO, MN, FMakokha, $\mathrm{PM}, \mathrm{RM}, \mathrm{DN}$ and MK will help in data collection. MN and MK will be involved in data analysis. MK wrote the first draft of the manuscript. All authors reviewed and approved the protocol.

Funding This work was supported by The Kenya National Research Fund (grant number NRF/MKU/2017/007 to JG).

Competing interests None declared.

Patient and public involvement Patients and/or the public were not involved in the design, or conduct, or reporting, or dissemination plans of this research.

Patient consent for publication Not required.

Provenance and peer review Not commissioned; externally peer reviewed.

Open access This is an open access article distributed in accordance with the Creative Commons Attribution Non Commercial (CC BY-NC 4.0) license, which permits others to distribute, remix, adapt, build upon this work non-commercially, and license their derivative works on different terms, provided the original work is properly cited, appropriate credit is given, any changes made indicated, and the use is non-commercial. See: http://creativecommons.org/licenses/by-nc/4.0/.

ORCID iD

Moses Kamita http://orcid.org/0000-0002-1756-932X 


\section{REFERENCES}

1 Aslam B, Wang W, Arshad MI, et al. Antibiotic resistance: a rundown of a global crisis. Infect Drug Resist 2018;11:1645-58.

2 Livermore DM. Has the era of untreatable infections arrived? J Antimicrob Chemother 2009;64 Suppl 1:i29-36.

3 Bejon P, Mwangi I, Ngetsa C, et al. Invasive gram-negative bacilli are frequently resistant to standard antibiotics for children admitted to hospital in Kilifi, Kenya. J Antimicrob Chemother 2005;56:232-5.

4 Kariuki S, Revathi G, Kiiru J, et al. Typhoid in Kenya is associated with a dominant multidrug-resistant Salmonella enterica serovar typhi haplotype that is also widespread in Southeast Asia. J Clin Microbiol 2010;48:2171-6.

5 WHO. United nations meeting on antimicrobial resistance, 2016.

6 Wellington $\mathrm{EMH}$, Boxall $\mathrm{AB}$, Cross $\mathrm{P}$, et al. The role of the natural environment in the emergence of antibiotic resistance in gramnegative bacteria. Lancet Infect Dis 2013;13:155-65.

7 Roca I, Akova M, Baquero F, et al. The global threat of antimicrobial resistance: science for intervention. New Microbes New Infect 2015;6:22-9.

8 Gudiol C, Tubau F, Calatayud L, et al. Bacteraemia due to multidrug-resistant gram-negative bacilli in cancer patients: risk factors, antibiotic therapy and outcomes. J Antimicrob Chemother 2011;66:657-63.

9 Gyssens IC, Kern WV, Livermore DM, et al. The role of antibiotic stewardship in limiting antibacterial resistance among hematology patients. Haematologica 2013;98:1821-5.

10 Vialle-Valentin CE, LeCates RF, Zhang F, et al. Predictors of antibiotic use in African communities: evidence from medicines household surveys in five countries. Trop Med Int Heal 2012;17:211-22.

11 Paruk F, Richards G, Scribante J, et al. Antibiotic prescription practices and their relationship to outcome in South Africa: findings of the prevalence of infection in South African intensive care units (Pisa) study. S Afr Med J 2012;102:613-6.

12 Ingram PR, Seet JM, Budgeon CA, et al. Point-Prevalence study of inappropriate antibiotic use at a tertiary Australian hospital. Intern Med J 2012:42:719-21.

13 Fridkin S, Baggs J, Fagan R, et al. Vital signs: improving antibiotic use among hospitalized patients. MMWR Morb Mortal Wkly Rep 2014;63:194-200.

14 Ayukekbong JA, Ntemgwa M, Atabe AN. The threat of antimicrobial resistance in developing countries: causes and control strategies. Antimicrob Resist Infect Control 2017:6:1-8.

15 Kariuki S, Revathi G, Kariuki N, et al. Increasing prevalence of multidrug-resistant non-typhoidal salmonellae, Kenya, 1994-2003. Int J Antimicrob Agents 2005;25:38-43.

16 Global Antibiotic Resistance Partnership - Kenya Working. Situational analysis and recommendations on antimicrobial use and resistance in Kenya. Center for Disease Dynamics, Economics \& Policy, 2011.

17 Gerding DN. The search for good antimicrobial stewardship. Jt Comm J Qual Improv 2001;27:403-4.
18 Drummond M, Jönsson B. Moving beyond the drug budget silo mentality in Europe. Value Health 2003;6:S74-7.

19 Malani AN, Richards PG, Kapila S, et al. Clinical and economic outcomes from a community hospital's antimicrobial stewardship program. Am J Infect Control 2013;41:145-8.

20 Dellit TH, Owens RC, McGowan JE, et al. Infectious diseases Society of America and the Society for healthcare epidemiology of America guidelines for developing an institutional program to enhance antimicrobial stewardship. Clin Infect Dis 2007;44:159-77.

21 Bogaert D, van Belkum A. Antibiotic treatment and stewardship in the era of microbiota-oriented diagnostics. Eur J Clin Microbiol Infect Dis 2018;37:795-8.

22 Glasgow RE, Vogt TM, Boles SM. Evaluating the public health impact of health promotion interventions: the RE-AIM framework. Am J Public Health 1999;89:1322-7.

23 World Health Organization. Essential medicines and health products, 2019. Available: https://www.who.int/medicines/regulation/ medicines-safety/toolkit_indicators/en/index2.html [Accessed 21st Sep 2019].

24 CLSI. Performance standards for antimicrobial susceptibility testing, 2019.

25 Karanika S, Paudel S, Grigoras C, et al. Systematic review and metaanalysis of clinical and economic outcomes from the implementation of hospital-based antimicrobial stewardship programs. Antimicrob Agents Chemother 2016;60:4840-52.

26 Borde JP, Nussbaum S, Hauser S, et al. Implementing an intensified antibiotic stewardship programme targeting daptomycin use in orthopaedic surgery: a cost-benefit analysis from the hospital perspective. Infection 2016;44:301-7.

27 WHO. WHO | Metrics: Disability-Adjusted Life Year (DALY). WHO 2014. Available: https://www.who.int/healthinfo/global_burden disease/metrics_daly/en/ [Accessed 25th Feb 2019]

28 Duane S, Domegan C, Callan A, et al. Using qualitative insights to change practice: exploring the culture of antibiotic prescribing and consumption for urinary tract infections. BMJ Open 2016;6:e008894.

29 Kauffmann RMet al. Infection reduction strategies including antibiotic stewardship protocols in surgical and trauma intensive care units are associated with reduced resistant gram-negative healthcareassociated infections. Surg. Infect. 2010;12:15-25.

30 Yong MK, Buising KL, Cheng AC, et al. Improved susceptibility of gram-negative bacteria in an intensive care unit following implementation of a computerized antibiotic decision support system. J Antimicrob Chemother 2010;65:1062-9.

31 Odhiambo F, Boru W, Wences A, et al. Antimicrobial resistance: capacity and practices among clinical laboratories in Kenya, 2013. Pan Afr Med J 2014;19:332.

32 Barasa EW, Molyneux S, English M, et al. Setting healthcare priorities in hospitals: a review of empirical studies. Health Policy Plan 2015;30:386-96. 